\title{
A RELATIONAL MODELING SYSTEM FOR LINEAR AND INTEGER PROGRAMMING
}

\author{
A. ATAMTÜRK, E. L. JOHNSON, J. T. LINDEROTH, and M. W. P. SAVELSBERGH \\ School of Industrial and Systems Engineering, Georgia Institute of Technology, Atlanta, Georgia 30332-0205 \\ atamturk@ieor.berkeley.edu
}

(Received October 1996; revisions received April 1998, April 1999; accepted July 1999)

\begin{abstract}
We discuss an integer linear programming modeling system based on relational algebra. In this system, all modeling related activities, such as model formulation, model instantiation, and model and instance management, are done using simple operations such as selection, projection, and predicated join.
\end{abstract}

\section{INTRODUCTION}

Anyone who has ever attempted to apply mathematical programming in practice knows that it is usually not a simple and straightforward exercise. The road from a real-life problem situation to a satisfactory solution can be quite long and full of complications. There are many factors that contribute to this, but one of the most important is the large amount of data that needs to be handled.

Because it is standard practice in industry to store information in databases, Mitra et al. (1995) argue that for mathematical programming to gain better acceptance as a modeling tool within corporate decision studies, a unified approach that integrates a modeling language with a relational database is necessary because it will provide a more powerful tool for constructing models that are truly data driven. They propose to achieve this by incorporating relational database structures into the syntax of the algebraic modeling language MPL (Maximal Software 1993).

The goal of our research has been to design an integrated modeling environment in which data management plays an even more central role and in which all modeling activities, such as model formulation, model instantiation, model solution, model validation, and solution analysis, are done in a common paradigm. Our efforts have resulted in a modeling environment based on relational algebra. Model formulation, instance generation, and solution manipulation are all done using relational operators such as selection, projection, and join. Many other desirable features of modeling environments, such as model management, instance management, and report writing are facilitated because they can be done using available relational database tools. Furthermore, model builder as well as end-user can work with the same system and users can easily share models. A prototype has been implemented to provide a "proof of concept." The prototype demonstrates that it is possible to develop a modeling environment for mathematical programming using a single paradigm: relational algebra.

Several other researchers have observed the potential of relational algebra for mathematical programming modeling.
Our research was partly motivated by the ideas presented by Johnson (1989). Choobineh (1991) designed SQLMP, an extension of SQL (Structured Query Language) (ANS, Date 1987) for mathematical programming that uses the algebraic paradigm for model conceptualization. We do not extend SQL and use the block-schematic paradigm for model conceptualization. The block-schematic paradigm is described by Baker (1983) and Welch (1987) and forms the basis of MathPro (1989) and MIMI (Baker 1992). The reason we use the block-schematic paradigm is purely pragmatic: The block-schematic approach is easier to embed in a relational modeling scheme. We are not claiming that the block-schematic paradigm is better than the algebraic approach used in systems such as GAMS (Brooke et al. 1988), AMPL (Fourier et al. 1993), MPL (Maximal Software 1993) and AIMMS (Bisschop and Entriken 1993). Which approach to use is largely a matter of taste, although it is claimed that many industrial users, particularly those in the process industry, prefer the block-schematic paradigm as it is closer to their "activity-based" view of the model. One disadvantage of the block-schematic paradigm is that it is mainly appropriate for linear optimization models. Dolk (1988) shows how structured models (as introduced by Geoffrion, 1987) can be represented and manipulated easily using SQL. Dolk also discusses how SQL might be used to facilitate the solution of mathematical programming models. He expects that this will require nonstandard SQL features. Our research shows that interfacing with an optimizer can be done completely with the tools used to access a relational database system. By using a standard interface language like SQL, the interface can be represented in a database-independent way, with some minor exceptions.

The paper is organized as follows. In $\S 2$, we present our view on the characteristics of good modeling environments. In $\S 3$, we give a brief introduction to relational operators. In $\S 4$, we introduce the basic concepts of relational modeling. In $\S 5$, we show how to use these basic concepts to model the fleet assignment problem. In $\S 6$, we illustrate how these concepts can be implemented in a standard database

Subject classifications: Information systems: analysis and design. Philosophy of modeling. Area of review: COMPUTING \& DESIGN TECHNOLOGY. 
group all rows have the same value for the GROUP BY field. The SELECT clause is then applied to each group of the partitioned table rather than to each row of the original table.

EXAMPLE.

SELECT product, SUM (amount)

FROM demand

GROUP BY product;

The last SQL construct used in the design and implementation of our relational modeling system is the view. A view is simply a particular look at the database. Although a view is a table, it does not exist physically in the database as a table; no storage space or data are allocated for it. The CREATE VIEW statement is used to define a virtual table.

EXAMPLE.

CREATE VIEW largecap (plant, product, capacity) AS SELECT plant, product, capacity

FROM production

WHERE capacity $>1000$;

\section{RELATIONAL MODELING}

We will illustrate the basic concepts of relational modeling by means of an example. We consider a production distribution problem with single sourcing requirements (Mairs et al. 1978).

\subsection{Problem Situation}

The problem is to decide how much of each product to produce at plants, how to ship to warehouses and transship to demand centers, subject to the constraint that a warehouse has to ship all of the demand for all products to any demand center to which it ships. In other words, each demand center is assigned a single warehouse that must meet all its demand for the several products.

\subsection{Instance Data}

The data involved in this model are production cost per product per plant, production capacity per product per plant, shipping cost from plant to warehouse, shipping cost from warehouse to demand center, and demand per product per demand center. These data are assumed to be available in the database in user data tables: Production, Shipcost, Tranship, and Demand. An instance of the productiondistribution problem is given by the following user data tables. This instance will be used throughout our discussion of the basic concepts of our approach.

Table Production:

$\begin{array}{llll}\text { PLANT } & \text { PRODUCT } & \text { CAPACITY } & \text { COST } \\ ----- & ------ & ------- & --- \\ \text { topeka } & \text { chips } & 200 & 230 \\ \text { topeka } & \text { nachos } & 800 & 280 \\ \text { newyork } & \text { chips } & 600 & 255\end{array}$

Table Shipcost:

$\begin{array}{llr}\text { PLANT } & \text { WHSE } & \text { COST } \\ ------ & ------ & --- \\ \text { topeka } & \text { topeka } & 1 \\ \text { topeka } & \text { newyork } & 45 \\ \text { newyork } & \text { topeka } & 45 \\ \text { newyork } & \text { newyork } & 2\end{array}$

Table Tranship:

WHSE CENTER COST

-------- ------- ---

topeka east 60

topeka south 30

topeka west 40

newyork east 10

newyork south 30

newyork west 80

Table Demand:

$\begin{array}{llr}\text { CENTER } & \text { PRODUCT } & \text { AMOUNT } \\ \text {------ } & ------ & ----- \\ \text { east } & \text { chips } & 200 \\ \text { east } & \text { nachos } & 50 \\ \text { south } & \text { chips } & 250 \\ \text { south } & \text { nachos } & 180 \\ \text { west } & \text { chips } & 150 \\ \text { west } & \text { nachos } & 300\end{array}$

\subsection{Column and Row Strips}

In an integer linear program, activities or decisions are modeled as variables, possibly with integrality restrictions on some of them, and restrictions and relations among the decisions are modeled as linear equations and inequalities in terms of the variables. Typically, variables in an integer linear program can be grouped into classes with similar characteristics, based on what they represent in the underlying problem situation. Similarly, the linear equations and inequalities, or constraints, can also be grouped into classes with similar characteristics. These classes of variables and classes of constraints can be used to construct a block-schematic view of the integer linear program, see for instance Welch (1987). In a block-schematic view, classes of variables are called column strips, classes of constraints are called row strips, and their intersections, where interactions occur, are called blocks.

There are three types of decisions (classes of variables) in our production distribution model:

- How much to produce of each product at a plant?

- How much of each product to ship from a plant to a warehouse?

- Which warehouse to assign to each demand center?

Each type of decision will be represented by a class of variables in the model and by a column strip in the blockschematic representation. Because there are three types of decisions, there will be three column strips: Produce, Ship, and Assign. 
Because each decision is related to a specific subset of the data, e.g., we have to determine how much to produce for each combination of a plant and a product, we can think of column strips as selections of data, and we can thus define them using the SQL construct of a view.

Ultimately, a modeling system has to prepare a machinereadable form of an instance for an optimizer. Therefore, any modeling system has to create a representation of the coefficient matrix that is understood by the optimizer. This is typically done in the form of triplets, one for each nonzero coefficient, consisting of a column (or variable) index, a row (or constraint) index, and the value of the coefficient. To accommodate the generation of such a representation, we store an index with each variable and each constraint.

Because the relational database paradigm does not impose an ordering on the rows of a table, it is nontrivial to create such an index. Fortunately, most commercial implementations of SQL provide a pseudo-column that contains a number indicating the sequence in which a row was selected, and we will make use of this pseudocolumn to create our index. In Oracle this pseudo-column is called RowNum, in Informix it is called RowId, whereas in Microsoft Access it is called AutoNumber. Because this is not a standard SQL feature and different vendors use different names for this feature, our relational modeling system has to be adapted depending on the database management system used for the implementation. In the remainder we assume that we are working with Oracle and use the RowNum feature to define the unique indices for each row and column strip.

Throughout the paper, we carry the indices in the tables explicitly, because they are important to convey the underlying concepts of our relational modeling system. However, in a commercial implementation based on the relational concepts we present, these indices can be hidden from the user because their generation and manipulation can be done automatically.

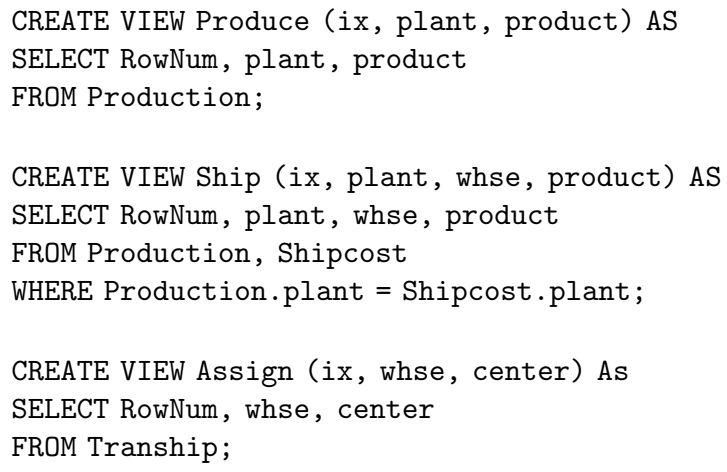

The definition of Ship, for example, indicates that there will be a variable for each combination of a plant, a warehouse, and a product and that these combinations can be obtained from the user data tables Production and Shipcost. Based on the data tables of the instance specified above, the column strip Ship defines the following variables plus associated indices.

$\begin{array}{llll}\text { IX } & \text { PLANT } & \text { WHSE } & \text { PRODUCT } \\ 1 & \text { topeka } & \text { topeka chips } \\ 2 & \text { topeka newyork chips } \\ 3 & \text { topeka } & \text { topeka nachos } \\ 4 & \text { topeka newyork nachos } \\ 5 & \text { newyork topeka chips } \\ 6 & \text { newyork newyork chips }\end{array}$

Similarly, there are three types of relations and restrictions in the model:

- Production at a plant is linked to shipping from the plant to a warehouse, i.e., everything that is produced should be shipped to some warehouse.

- Enforcement of the product flow balance, i.e., the total amount of a product shipped from plants to a warehouse should equal the total amount of a product shipped to the centers.

- Enforcement of the single sourcing requirement, i.e., each center receives all its demand from a single warehouse.

Each class of constraints will be represented by a row strip in the block-schematic representation. Because there are three classes of constraints, there will be three row strips: Prodrow, Shiprow, and Centrow.

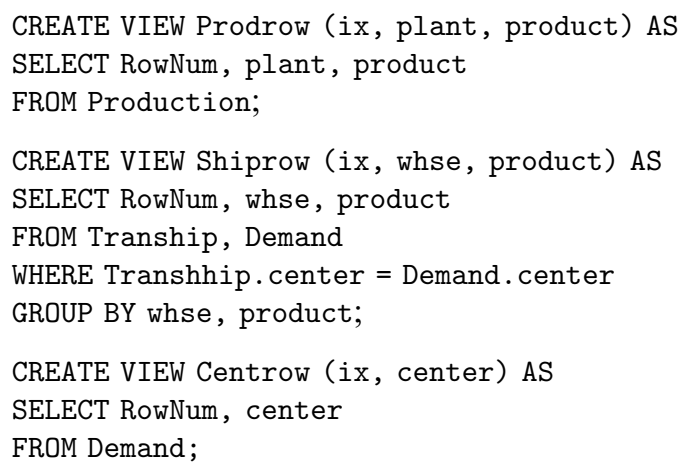

Based on the data tables of the instance specified above, the row strip ShipRow defines the following constraints plus associated indices:

\begin{tabular}{lll} 
IX & WAREHOUSE & PRODUCT \\
\hdashline & topeka & chips \\
2 & topeka & nachos \\
3 & newyork & chips \\
4 & newyork & nachos
\end{tabular}

Observe that we do not specify the number of variables in a class or the number of constraints in a class. The size of an instance is not part of the model but is determined automatically by the number of records in the user data tables.

\subsection{Blocks}

So far, we have defined the column strips and row strips of the matrix, i.e., the classes of variables and the classes of constraints of the model. Next, we have to determine 
whether a class of variables interacts with a class of constraints, i.e., whether there are nonzero entries in the block defined by the associated column and row strips. This gives the blocks with the technological coefficients of the matrix.

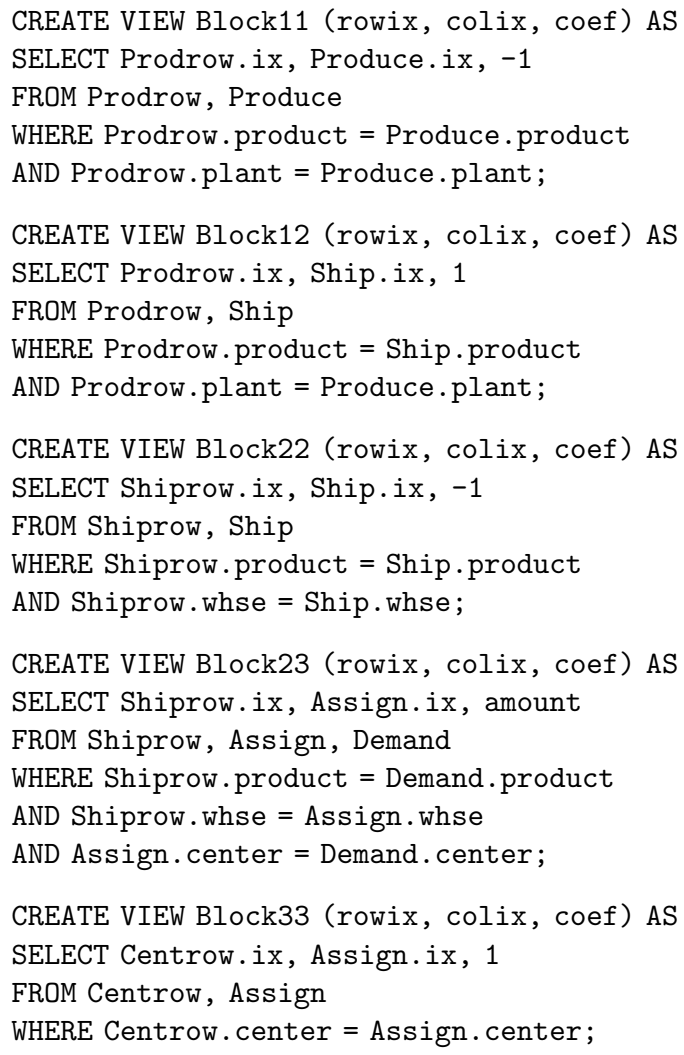

The definition of Block23, for example, indicates that there will be a nonzero coefficient for each product that is shipped from a warehouse to a demand center and that the value of this coefficient is equal to the demand at this demand center, which can be found in the user data table Demand.

As an example, we show the intermediate table that is implicitly generated during the construction of the virtual table Block23 just before the final selection of rowix, colix, and coef is made.

\begin{tabular}{lllrll} 
WHSE & PRODUCT & CENTER & COEF & COLIX & ROWIX \\
\hline topeka & chips & east & 200 & 1 & 1 \\
topeka & chips & south & 250 & 2 & 1 \\
topeka & chips & west & 150 & 3 & 1 \\
topeka & nachos & east & 50 & 1 & 2 \\
topeka & nachos & south & 180 & 2 & 2 \\
topeka & nachos & west & 300 & 3 & 2 \\
newyork chips & east & 200 & 4 & 3 \\
newyork chips & south & 250 & 5 & 3 \\
newyork chips & west & 150 & 6 & 3 \\
newyork nachos & east & 50 & 4 & 4 \\
newyork nachos & south & 180 & 5 & 4 \\
newyork nachos & west & 300 & 6 & 4
\end{tabular}

Observe that each block defines a set of triplets specifying the nonzero coefficients of that block, and that all triplets are specified relative to that block. Therefore, to specify the complete matrix all we have to do is impose an ordering on the column strips and row strips and add the appropriate offsets to the row and column indices appearing in the triplets.

It is convenient for us to consider information pertaining purely to a class of variables, such as objective coefficients, lower, and upper bounds, and information pertaining purely to a class of constraints, such as lower and upper bounds, as blocks as well. Because this type of information is typically referred to as belonging to the rim of the matrix, we will sometimes refer to these blocks as rim blocks. Note that we specify constraints using lower and upper bounds on the activity instead of using a sense and a right-hand side.

Below are the definitions of the rim blocks. Because these blocks will be part of the matrix description that will be input to an integer linear programming optimizer, we create triplets.

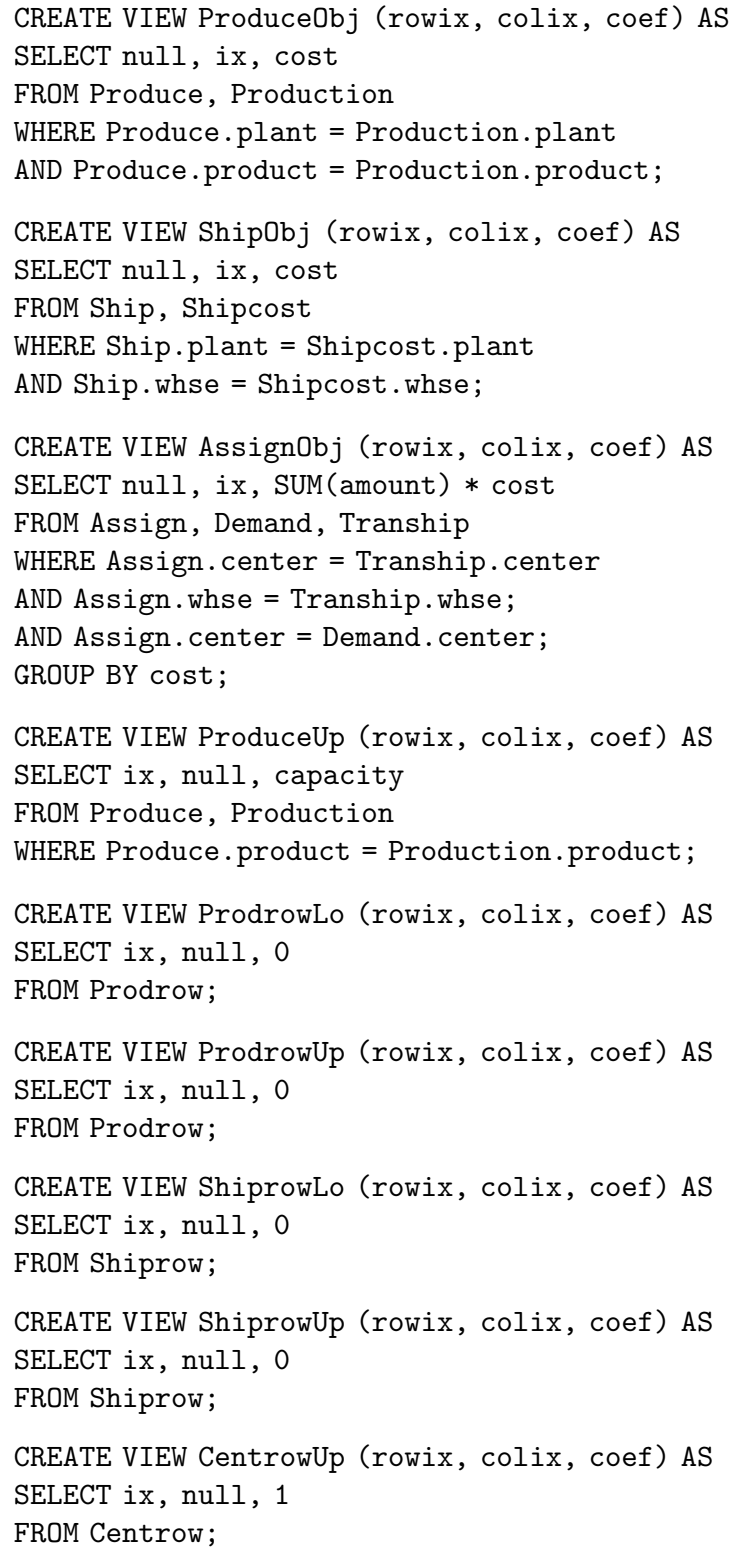


The definition of ProduceObj indicates that for each combination of a plant and a product defined in the column strip Produce, the objective coefficient can be found in the Production user data table in the field COST of the row that matches this particular combination. The definition of AssignObj shows that it is also possible to have computed objective coefficients. This completes the model description. A block-schematic view of the model is given here.

\begin{tabular}{|l|c|c|c|c|c|}
\hline & RowLo & Produce & Ship & Assign & RowUp \\
\hline Objective & & ProduceObj & Ship0bj & AssignObj & \\
ColumnLo & & 0 & 0 & 0 & \\
ProdRow & $0 \leqslant$ & Block11 & Block12 & & $\leqslant 0$ \\
ShipRow & $0 \leqslant$ & & Block22 & Block23 & $\leqslant 0$ \\
CentRow & $1 \leqslant$ & & & Block33 & $\leqslant 1$ \\
ColumnUp & & ProduceUp & Inf & Inf & \\
\hline
\end{tabular}

Observe that the definition of column strips, row strips, and blocks depends only on the structure of the user data tables, not on the records contained in those tables. This ensures complete separation of model and data. It also means that the same model definition can handle instances with two plants, two products, two warehouses, and three demand centers, as well as instances with hundreds of plants, thousands of products, hundreds of warehouses, and millions of demand centers. Observe that an instance of a model exists as a collection of views, i.e., virtual tables. This is a major difference from systems in which instances are physically stored in a database.

In defining the column strips, row strips, and blocks of the production distribution model, we have presented the required SQL statements in full detail. This was done to convey how a relational modeling system can be implemented. However, a lot of information contained in the SQL statements does not pertain to the model, but is a result of the SQL syntax. In any commercial implementation of a modeling environment based on relational modeling concepts, a user interface needs to be developed that shields a user from the underlying SQL syntax and indexing, reducing the effort required to specify a model. In such a system, the user would specify a block simply by using the names of the column and row strips and the corresponding relationship between the attributes of the strips. The actual detailed SQL queries would be created automatically by the system.

\subsection{Ordered Domains}

An important class of linear programming models involves multiperiod production planning. Such models typically contain a class of balancing constraints that ensure a proper transition from one period to the next, e.g., for every period except the first, the inventory at the start of period $t-1$ plus the production in period $t-1$ minus the sales in period $t-1$ has to equal the inventory at the start of period $t$. Such models pose a serious problem for the relational modeling approach because it relies on a natural ordering of the data, such as weeks, months, and years.
The relational model that forms the basis of relational database implementations does not support the concept of ordered domains. There are two ways to deal with this dilemma. First, commercial implementations of a relational database have special functions related to time and we could make use of these. Second, when building a model, we can use numerical representations of the ordered domains and use SQL constructs to implement ordering concepts such as "first," "successor," and "predecessor."

As an example, consider the following two user data tables. The first table is not necessary but mainly serves as a table that can be used in the report generation phase.

Table Date:

\begin{tabular}{ll} 
NAME & PERIOD \\
\hdashline$-\cdots-1$ & $-\cdots-1$ \\
February & 2 \\
April & 4 \\
June & 6
\end{tabular}

Table Production:

$\begin{array}{llll}\text { PRODUCT } & \text { PERIOD } & \text { CAPACITY } & \text { COST } \\ ----- & ----- & ----- & ---- \\ \text { chips } & 2 & 2000 & 76 \\ \text { chips } & 4 & 1600 & 78 \\ \text { chips } & 6 & 2000 & 76 \\ \text { nachos } & 2 & 1200 & 82 \\ \text { nachos } & 4 & 1200 & 82 \\ \text { nachos } & 6 & 800 & 86\end{array}$

Consider the class of balancing constraints mentioned above. The column strip associated with the inventory variables can be defined as

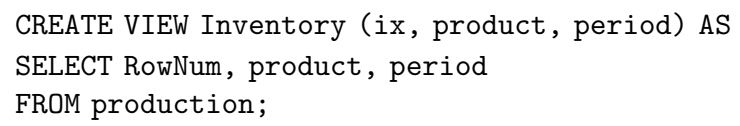

We require a balance constraint for each product in each period except for the first. The row strip associated with the balancing constraints can be defined as

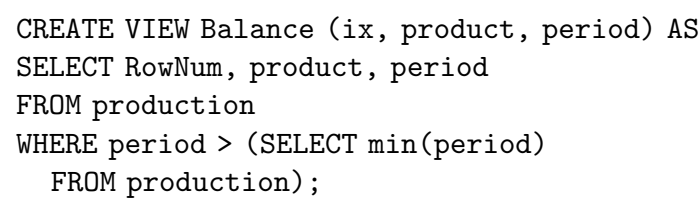

We have used a subquery to determine the first period appearing in the table production. For this class of constraints, there are two interactions between the column strip and the row strip - there is product flow "into" the period and "out" of the period. To accomplish this, we simply define the two matrix blocks:

CREATE VIEW Block_1 (rowix, colix, coef) AS

SELECT Balance.ix, Inventory.ix, -1

FROM Balance, Inventory

WHERE Balance . product $=$ Inventory . product

AND Balance $\cdot$ period = Inventory $\cdot$ period; 


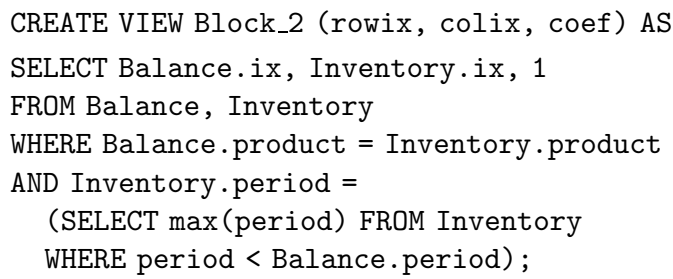

Again, we have used a subquery, this time to determine the previous period appearing in the production table. This concludes our description of the basic concepts of relational modeling. In Appendix A, we provide examples of relational models for some well-known planning problems.

\section{THE FLEET ASSIGNMENT PROBLEM}

To provide the reader with a real-life application in which using the relational modeling scheme is a natural and convenient choice, we discuss an important planning problem faced by the airline companies. In the fleet assignment problem, we are given a flight schedule and a set of fleet (aircraft) types. The problem is to find a minimum cost assignment of the fleet types to the flight legs in the schedule (see Abara 1989 for a discussion and overview of the fleet assignment problem). The flight schedule (time table) of a medium to large airline is huge and is typically stored in a relational database. Below we show some of the typical information found in the database.

Table Schedule:

\begin{tabular}{|c|c|c|c|c|c|c|}
\hline LEG & DEPSTA & DEPTIM & ARRSTA & ARRTIM & FLEET & COST \\
\hline 101 & DFW & 745 & BOS & 1055 & 734 & 8270 \\
\hline 101 & DFW & 745 & BOS & 1055 & 757 & 11088 \\
\hline 101 & $\mathrm{DFW}$ & 745 & BOS & 1055 & 767 & 12098 \\
\hline 102 & BOS & 1200 & $\mathrm{DFW}$ & 1500 & 734 & 8270 \\
\hline 102 & BOS & 1200 & $\mathrm{DFW}$ & 1500 & 757 & 11088 \\
\hline 102 & BOS & 1200 & $\mathrm{DFW}$ & 1500 & 767 & 12098 \\
\hline 201 & $\mathrm{DFW}$ & 745 & SFO & 1145 & 734 & 9653 \\
\hline
\end{tabular}

In this particular example, we see that there is flight from Dallas/Fort Worth International Airport to Logan Airport in Boston departing at 7:45 $\mathrm{AM}$ and arriving at 10:55 $\mathrm{AM}$ and that this flight can be flown by either a Boeing 734 at a cost of 8270, a Boeing 757 at a cost of 11088 , or a Boeing 767 at a cost of 12098 .

The information on available fleet types is also stored in a relational database and looks somewhat like this:

Table Fleets:

$\begin{array}{lll}\text { FLEET } & \text { AVAIL } & \ldots \\ ---- & --- & \\ 734 & 6 & \ldots \\ 757 & 2 & \ldots \\ 767 & 9 & \ldots \\ \ldots & & \end{array}$

A feasible fleet assignment must assign a fleet type to each flight leg, cannot use more aircrafts of a given fleet type than are available, and must ensure that aircrafts that arrive at a station either depart or stay on the ground and similarly that aircrafts depart from a station where they landed earlier.

To model the fleet assignment problem, we introduce two classes of variables: (1) a class of binary variables indicating for each combination of flight leg and fleet type whether or not the fleet type is assigned to the flight leg, and (2) a class of integer variables counting the number of aircrafts of a specific fleet type on the ground at a particular station and time.

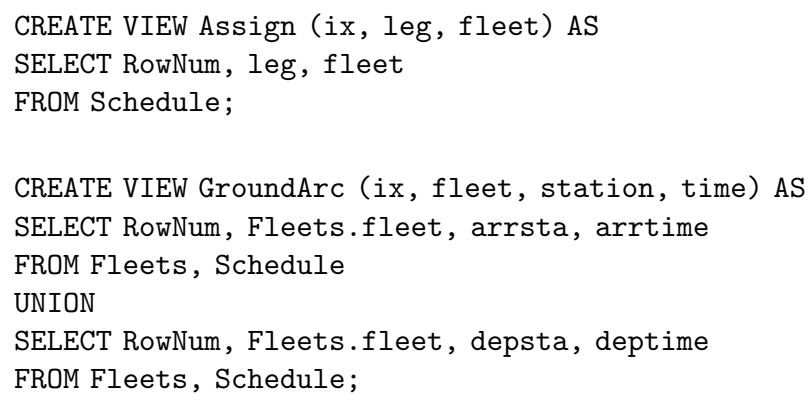

Note that we create a so-called ground arc for each fleet type at each station and each event (arrival or departure) at that station. Ground arcs, as the name suggests are used to model aircrafts that stay on the ground at a station.

There are three classes of constraints. First, which is at the heart of the problem, we have to ensure that each flight leg is assigned exactly one fleet type.

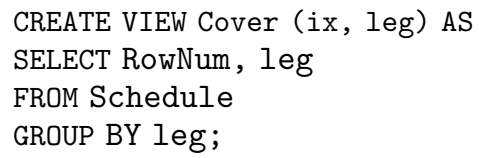

Second, we have to ensure that the fleet assignments are consistent, in the sense that there is flow balance for each fleet type at each station and each event occurring at that station. That is to say that for each fleet type, the number of aircrafts of that fleet type "arriving" at a station (either via an incoming flight leg or via a ground arc) is equal to the number of aircrafts "departing" from the station (either via an outgoing flight leg or a ground arc).

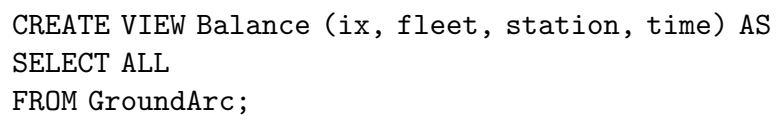

Finally, we have to ensure for each fleet type that we do not use more aircrafts than there are available.

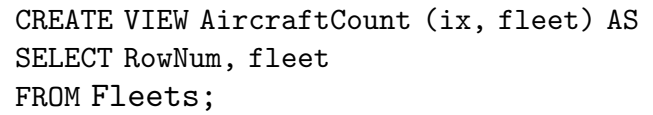

Now we define the blocks of the matrix. Because we have to assign exactly one fleet type to each flight leg, we have 
the following block

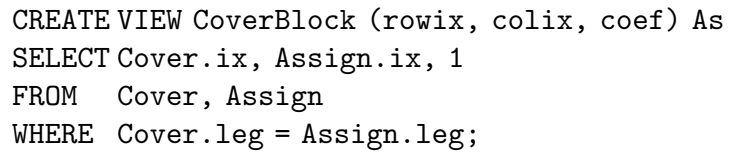

Next, we consider the blocks that define the flow balance constraints for each fleet type at each event occurring at a station. This involves selecting appropriate incoming and outgoing arcs. We create a block handling the outgoing flight legs first.

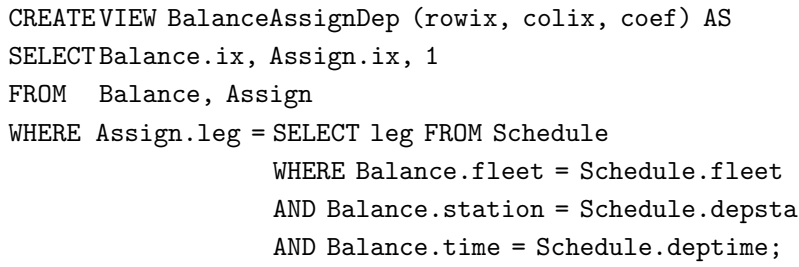

Next, we create a block handling the incoming flight

CREATE VIEW BalanceAssignArr (rowix, colix, coef) AS

SELECT Balance.ix, Assign.ix, -1

FROM Balance, Assign

WHERE Assign.leg = SELECT leg FROM Schedule

WHERE Balance.fleet $=$ Schedule.fleet

AND Balance.station $=$ Schedule. arrsta

AND Balance.time $=$ Schedule.arrtime;

Now, we switch to ground arcs and start with outgoing ground arcs.

CREATE VIEW BalanceGroundOut (rowix, colix, coef) AS

SELECT Balance.ix, Ground.ix, 1

FROM Balance, Ground

WHERE Balance.fleet $=$ Ground. fleet

AND Balance.station $=$ Ground. station

AND Balance.time $=$ Ground.time;

We follow with the incoming ground arcs. Selecting the incoming arcs is a bit more involved, since it requires the identification of the previous event at a station, which is done by means of a subquery. Furthermore, we need to distinguish the first event at a station from the other events because the previous event of the first event actually occurs as the last event occurring (of the previous day).

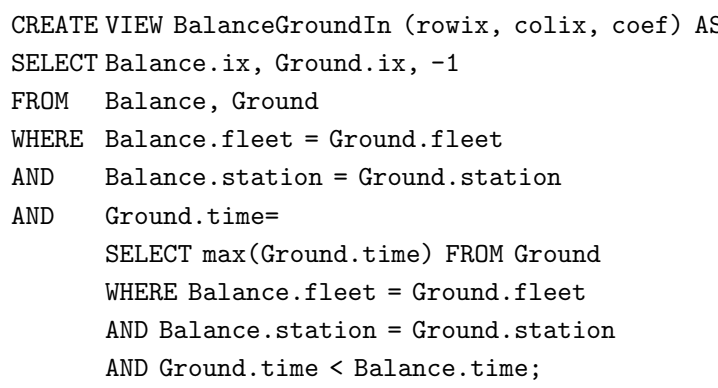

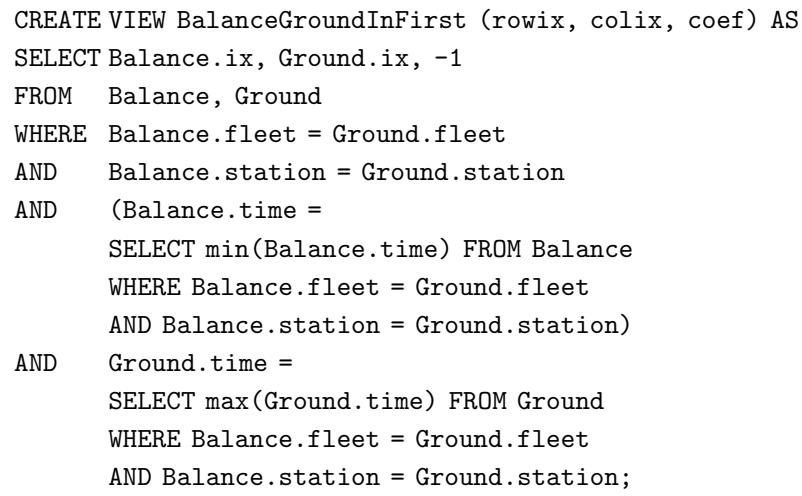

To ensure for each fleet type that we do not use more aircrafts than there are available, we take a snapshot at midnight and count all the aircrafts of a specific fleet type. Because the balancing constraints ensure that the flow is a circulation the number of aircrafts in use will be the same throughout the day and taking a snapshot at midnight suffices. There are two blocks: one to account for the "red eye" flights, i.e., flight legs corresponding to flights that are in the air at midnight, and one for the ground arcs that cross midnight.

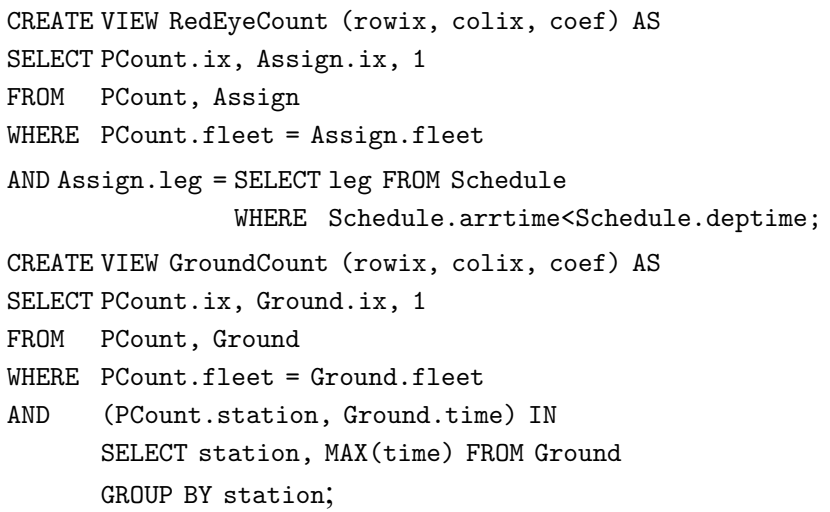

Specifying the rim blocks, i.e., objective function coefficients and lower and upper bounds on variables and constraints is straightforward.

\section{A RELATIONAL MODELING SYSTEM}

The preceding sections have shown the conceptual viability of modeling linear and integer programs using a relational scheme. In this section, we describe the design of a modeling environment that supports the relational modeling paradigm. More specifically, we show how to implement model management, instance management, and solver management using relational database tools. These management tasks are performed by the modeling environment and are typically invisible to a user of the system.

\subsection{Model Management}

In the block schematic approach, a mathematical programming model is specified entirely in terms of row strips, column strips, and matrix blocks. In a relational database environment we can conveniently manage this information 
for many models. We create four "system" tables: SysModels, SysRows, SysCols, and SysBlocks that contain all the information about the views defining the various models.

The SysModels table contains the names of the models present in the system. It has attributes Model and ObjSense. The Model attribute is the unique name of a model and the ObjSense attribute is MAX or MIN indicating whether the specified model is a maximization or minimization problem.

The SysRows table contains the names of the row strips present in the system. It has attributes Model and RowStrip. The Model attribute is the unique name of a model and the RowStrip attribute is the unique name of a view defining a row strip of the specified model.

The SysCols table contains the names of the column strips present in the system. It has attributes Model, RowStrip, and Type. The Model attribute is the unique name of a model, the ColStrip attribute is the unique name of a view defining a column strip of the specified model, and the Type attribute is CONTINUOUS, BINARY, or INTEGER indicating the variable type associated with the specified column strip.

The SysBlocks table contains the names of the blocks present in the system. It has attributes Model, Block, RowStrip, ColStrip, and Type. The Model attribute is the unique name of a model, the Block attribute is the unique name of a matrix block of the specified model, the RowStrip attribute is the unique name of the view defining the row strip associated with the specified block, the ColStrip attribute is the unique name of the view defining the column strip associated with the specified block, and the Type attribute is ROWLOWER, ROWUPPER COLOBJ, COLLOWER, COLUPPER, or BLOCKDATA indicating the type of the specified block.

The tables below show the relevant entries in the system tables pertaining to the production distribution model presented in the preceding sections.

Table SysModels:

Model ObjSense

Prod-Dist MIN

... ...

Table SysRows :

Model RowStrip

Prod-Dist Prodrow

Prod-Dist Shiprow

Prod-Dist Centrow

... . .

Table SysCols:

$\begin{array}{lll}\text { Model } & \text { RowStrip } & \text { Type } \\ \text {---- } & ----- & ------ \\ \text { Prod-Dist } & \text { Produce } & \text { CONTINUOUS } \\ \text { Prod-Dist } & \text { Ship } & \text { CONTINUOUS } \\ \text { Prod-Dist } & \text { Assign } & \text { BINARY } \\ \ldots & \ldots & \ldots\end{array}$

Table SysBlocks:

$\begin{array}{lllll}\text { Model } & \text { Block } & \text { RowStrip } & \text { ColStrip } & \text { Type } \\ \text {----- } & ----- & ----- & ----- & ----- \\ \text { Prod-Dist } & \text { Block11 } & \text { Prodrow } & \text { Produce } & \text { BLOCKDATA } \\ \text { Prod-Dist } & \text { Block12 } & \text { Prodrow } & \text { Ship } & \text { BLOCKDATA } \\ \text { Prod-Dist } & \text { Block22 } & \text { Shiprow } & \text { Ship } & \text { BLOCKDATA } \\ \text { Prod-Dist } & \text { Block23 } & \text { Shiprow } & \text { Assign } & \text { BLOCKDATA } \\ \text { Prod-Dist } & \text { Block33 } & \text { Centrow } & \text { Assign } & \text { BLOCKDATA } \\ \text { Prod-Dist } & \text { ProduceObj } & & \text { Produce } & \text { COLOBJ } \\ \text { Prod-Dist } & \text { ShipObj } & & \text { Ship } & \text { COLOBJ } \\ \text { Prod-Dist } & \text { AssignObj } & & \text { Assign } & \text { COLOBJ } \\ \text { Prod-Dist } & \text { ProduceUp } & & \text { Produce } & \text { COLUPPER } \\ \text { Prod-Dist } & \text { ProdrowUp } & \text { Prodrow } & & \text { ROWUPPER } \\ \text { Prod-Dist } & \text { ProdrowUp } & \text { Prodrow } & & \text { ROWLOWER } \\ \text {... } & \ldots & \ldots & \ldots & \ldots\end{array}$

\subsection{Instance Management}

In the production distribution problem used to illustrate the relational modeling scheme, we have used specific data tables in the definition of the model-e.g., Production, Shipcost, Tranship, and Demand-even though we used only the structure of these tables. It is good practice, however, to define models completely independent of its instances. To do so, we make use of another feature of $\mathrm{SQL}$ called a synonym. A synonym is an alias assigned to a table or view that may thereafter be used to refer to it. For each data table required in the definition of a model, we introduce a synonym and all references to data tables are made through these synonyms. Then, to create a specific instance of a model, all that needs to be done is to update the synonyms so that they refer to the actual data tables defining the instance.

The relational database environment is well suited to manage many instances of the same model. We create two system tables: SysDataTables and SysInstances.

The SysDataTables table contains the names of the active user data tables for a model. It has attributes Model, BaseTable, Syn, and ActiveTable. The Model attribute is the unique name of a model, the Block attribute is the unique name of a matrix block, the BaseTable attribute is the unique name of a special data table, called base table, having the same structure as an instance data table required in the definition of the specified model, the Syn attribute is the synonym for the base table used in the model definition, and the ActiveTable attribute is the name of the current data table associated with the specified synonym.

As mentioned before, the definition of a model depends only on the structure of the user data tables, not on the records contained in those tables. Therefore, to completely separate model and data, we use artificial tables in the model definition. The artificial tables, which we call base tables, have the same structure as the user tables, but will always be empty. The use of base tables also gives the system a level of error-checking. When we attempt to associate a synonym with a user specified instance data table, we can check if this table has the proper structure by comparing it to the base table associated with the synonym. 
In our production distribution example, instead of using the tables Production, ShipCost, Tranship, and Demand directly in the definition of the model, we create (empty) base tables base_Production, base_ShipCost, base_Tranship, and base_Demand (with the same structure) and synonyms syn_Production, syn_ShipCost, syn_Tranship, and syn_Demand (initially pointing to the base tables), and use the synonyms in the definition of the model. Then to instantiate the model, we let the synonyms point to the real data tables.

\begin{tabular}{llll}
\multicolumn{2}{l}{ Table SysDataTables: } & & \\
Model & BaseTable & Syn & ActiveTable \\
----- & ------ & ------- & ------ \\
Prod-Dist & base_Production & syn_Production & Production \\
Prod-Dist & base_ShipCost & syn_ShipCost & ShipCost \\
Prod-Dist & base_Tranship & syn_Tranship & Tranship \\
Prod-Dist & base_Demand & syn_Demand & Demand
\end{tabular}

The SysInstances table contains the names of the (user data) tables of an instance. It has attributes Model, Instance, Syn, and DataTable. The Model attribute is the unique name of a model, the Instance attribute is a unique name of an instance of the specified model, the Syn attribute is the name of the synonym used in the definition of the specified model, and the DataTable attribute is the name of the data table associated with the specified synonym in the specified instance.

Table SysInstances:

$\begin{array}{llll}\text { Model } & \text { Instance } & \text { Syn } & \text { DataTable } \\ ----- & ------ & ------- & ------- \\ \text { Prod-Dist } & \text { PD_January } & \text { syn_Production } & \text { Jan_Production } \\ \text { Prod-Dist } & \text { PD_January } & \text { syn_ShipCost } & \text { ShipCost } \\ \text { Prod-Dist } & \text { PD_January } & \text { syn_Tranship } & \text { Tranship } \\ \text { Prod-Dist } & \text { PD_January } & \text { syn_Demand } & \text { Jan_Demand } \\ \text { Prod-Dist } & \text { PD_February } & \text { syn_Production } & \text { Feb_Production } \\ \text { Prod-Dist } & \text { PD_February } & \text { syn_ShipCost } & \text { ShipCost } \\ \text { Prod-Dist } & \text { PD_February } & \text { syn_Tranship } & \text { Tranship } \\ \text { Prod-Dist } & \text { PD_February } & \text { syn_Demand } & \text { Feb_Demand } \\ \text {... } & \ldots & \ldots & \ldots\end{array}$

Finally, there are two system tables that contain solution information: SysRuns and SysSols. The SysRuns table has attributes Model, Instance, Solver, Rundate, RunTime, $\mathrm{Obj}$, and CpuTime. The Model attribute is the unique name of a model, the Instance attribute is a unique name of an instance of the specified model, the Solver attribute indicates the solver used for the run, the RunDate attribute is the date of a particular run of the specified model for the specified instance, the RunTime attribute is the time of day that the run started, the $\mathrm{Obj}$ attribute is the objective function value obtained in the run, and the CpuTime attribute is the CPU time for the run.

Table SysRuns:

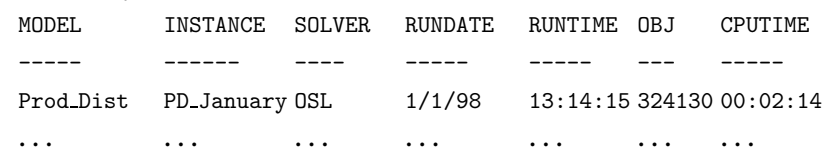

The SysSols table has attributes Model, Instance, RunDate, RunTime, StripName, and TableName. The Model attribute is the unique name of a model, the Instance attribute is a unique name of an instance of the specified model, the RunDate attribute is the date of a particular run of the specified model for the specified instance, the RunTime attribute is the time of day of the run, the StripName attribute is a row or a column strip name of the specified model, and the TableName attribute is the name of the data table containing the solution information for the specified row or column strip obtained in the run.

Table SysSols:

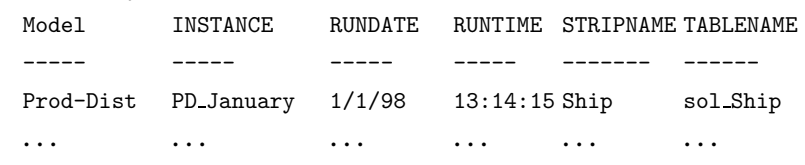

Table sol_Ship:

$\begin{array}{lllc}\text { PLANT } & \text { WHSE } & \text { PRODUCT } & \text { VALUE } \\ \text { topeka } & \text { topeka } & \text { chips } & 200 \\ \text { topeka } & \text { newyork } & \text { chips } & 0 \\ \text { topeka } & \text { topeka } & \text { nachos } & 480 \\ \text { topeka } & \text { newyork } & \text { nachos } & 50 \\ \text { newyork } & \text { topeka } & \text { chips } & 200 \\ \text { newyork } & \text { newyork } & \text { chips } & 200\end{array}$

Observe that the solution is put in a collection of tables. It is also possible, and in fact very easy, to put the solution immediately into the appropriate user data tables. We have chosen for the above design because it is more flexible and puts control in the hands of the user.

When the values of the decision variables have been returned to the user data tables, SQL provides a convenient tool for viewing the results of the optimization. In particular, one can easily scan subsets of the solution which may be of interest. For example, the production facility manager in Topeka can easily determine his production requirements and the total production cost by the following two queries (where we assume that the levels of production determined by the optimizer, i.e., the values of Produce, have been put in an additional field amount in the data table Production).

SELECT product, amount
FROM production
WHERE plant = 'topeka';
SELECT SUM(cost*amount)
FROM production
WHERE plant = 'topeka';

\subsection{Solver Management}

Another feature of the relational modeling environment that is easily incorporated is to allow users to vary solver parameters by defining a table SysParams to hold these parameters.

The SysParams table has attributes Solver, Parameter, and Value. The Solver attribute is the name of a solver, the 
Parameter attribute is the name of a parameter that can be set for the specified solver, and Value is the current value of the parameter.

\begin{tabular}{|c|c|c|}
\hline Solver & Parameter & Value \\
\hline CPLEX & CPX_PARAM_CLIQUES & 1 \\
\hline CPLEX & CPX_PARAM_NODELIM & 1000000 \\
\hline OSL & rtolpinf & 0.00001 \\
\hline & $\ldots$ & $\cdots$ \\
\hline
\end{tabular}

The system tables introduced above form the basis of the prototype relational modeling system ARMOS described in the next section. It should be noted that the system tables are created only once at the installation of the system and then used by the system as an internal database of existing models, instances, and solutions. Maintaining the system tables is a responsibility of the system, not of the user of the system.

\subsection{A Prototype}

To demonstrate the viability of the ideas and concepts presented in the previous sections, we have developed a small prototype system called ARMOS, A Relational Modeling System. ARMOS offers a simple user interface that is coded in Embedded SQL (Oracle Corp. 1992) and that allows a user to list the models stored in system, to load a model, to list the instances stored in the system for the loaded model, to make an instance active, to optimize the active instance, and to display solution values. The user can also display the model's matrix block structure, and view the coefficients of any particular matrix block of an active instance. Currently, both linear and mixed integer linear programs can be solved. ARMOS is built on top of the commercial software package OSL [DSV]. A brief description of its functionality can be found in Appendix B.

As mentioned above, ARMOS is a prototype. It provides only the most basic functionality and it only has a simple text-based user instance. There is no dedicated graphical editor supporting model development. Our goal in developing ARMOS was to verify the viability of using the relational modeling scheme in an actual implementation.

\section{DISCUSSION}

This paper provides a "proof of concept" demonstration, showing that it is possible to develop a modeling environment for mathematical programming using a single paradigm: relational algebra. We feel there are several advantages to such an approach. It is often observed, see for example Hürliman (1991) that despite recent developments, mathematical programming is still not fully exploited in practice. By designing a modeling environment centered around database management systems that are widely used in industry, we believe it is possible to make mathematical programming accessible to a wider audience. Furthermore, it is easy to set things up in such a way that using a model has a "fill-in-the-blank" feel, where solution values are immediately imported into the appropriate data tables. This will be very appealing to end-users, and will increase their acceptance level. (We have all witnessed the acceptance of spread-sheet like interfaces!) It has also been observed, see for example Mitra et al. (1995), that the data in corporate information systems are often regularly revised and that it is therefore desirable that a decision-making system monitors changes in instance data, notifies users that the current solution values are out of date, and allows users to update that solution value with a single command. Because in the relational modeling system we propose an instance exists only through virtual links rather than physically, this feature is naturally available.

Finally, a few words on how the proposed relational modeling system compares to other systems based on the block-schematic model building paradigm, such as MIMI and MathPro. Obviously, both MIMI and MathPro are, at the moment, far easier to use than our prototype system because of their more sophisticated graphical user interfaces. MIMI also has a much larger functionality, because it includes an expert system component for rule-based model solution. On the other hand, MIMI supports only two-dimensional tables and therefore requires a hierarchy of tables to represent high-dimensional strips or blocks. Our prototype does not impose this restriction because SQL easily handles tables with multiple fields. Furthermore, MIMI requires all relevant data to be in its own private internal database, which requires copying/transferring data from the corporate database to MIMI's database. Because the relational modeling system is built on top of the corporate database, it eliminates the data transfers and storage duplication.

\section{Appendix}

Appendices A and B can be found at the Operations Research Home Page: 〈http://or.pubs.informs.org/opsresearch.html $\rangle$ in the Online Collection.

\section{ACKNOWLEDGEMENTS}

The authors thank the area editor, the associate editor, and the referees for many helpful suggestions that significantly improved the quality and presentation of the paper. This research was supported, in part, by NSF Grant DMI-9700285 to the Georgia Institute of Technology.

\section{REFERENCES}

Abara, J. 1989. Applying integer linear programming to the fleet assignment problem. Interfaces 19 20-28.

Atamtürk, A., E. L. Johnson, J. T. Linderoth, M. W. P. Savelsbergh. 1996. Using ARMOS, a relational modeling system.

ANSI. 1986. Database Language SQL, Document ANSI X3. 135-1986. (Also available as ISO document ISO/TC97/ SC21/WG3 N117.) 
Baker, T. E. 1983. RESULT: An interactive modeling systems for planning and scheduling. Presented at the ORSA/TIMS meeting, Chicago, IL.

—. 1992. MIMI/LP User Manual. Chesapeake Decision Science, Inc.

Bisschop J., R. Entriken, 1993. AIMMS The Modeling System. Paragon Decision Technology.

Brooke, A., D. Kendrick, A. Meeraus. 1988. GAMS, A User's Guide. The Scientific Press, Redwood City, CA.

Choobineh, J. 1991. SQLMP: A data sublanguage for representation and formulation of linear mathematical models. ORSA J. Comput. 3(4) 358-375.

Date, C. 1987. A Guide to the SQL Standard. Addison/Wesley, Reading, MA.

Dolk, D. R. 1988. Model management and structured modeling: The role of an information resource dictionary system. Comm. ACM 31(6) 704-718.

Druckerman, J., D. Silverman, K. Viaropulos. Optimization Subroutine Library Release 2 Guide and Reference. IBM.

Elmasri, R., S. Navathe. 1994. Fundamentals of Data Base Systems, 2nd ed, Benjamin/Cummings.

Fourer, R., D. M. Gay, B. W. Kernighan. 1993. AMPL. A Modeling Language for Mathematical Programming. The Scientific Press, Redwood City, CA.

Geoffrion, A. M. 1987. An introduction to structured modeling. Management Sci. 35(5) 547-588.
Greenburg, H. I., F. H. Murphy. 1992. A comparison of mathematical programming modeling systems. Ann. Oper. Res. 38 177-238.

Hürlimann, T. 1991. Linear modeling tools. Working Paper 187, Institute for Automation and Operations Research, University of Fribourg, Switzerland.

Johnson, E. L. 1989. Modeling and strong linear programs for mixed integer programming. S. W. Wallace, ed. Algorithms and Model Formulations in Mathematical Programming. Springer-Verlag, Berlin, NATO ASI Series, Vol. F51, 1-43.

MathPro, Inc. 1989. MathPro Usage Guide: Introduction and Reference.

Maximal Software. 1993. MPL Modeling System.

Mitra, G. B., Kristjansson, C. Lucas, S. Moody. 1995. Sets and indices in linear programming modelling and their integration with relational data models. Comput. Optim. Appl. 4 263-292.

Mairs, T. G., G. W. Wakefield, E. L. Johnson, K. Speilbergh. 1978. On a production allocation and distribution problem. Management Sci. 24 1622-1630.

Oracle Corporation. 1992. Programmer's Guide to the ORACLE Precompilers, Version 1.5.

Wagner, H. 1975. Principles of Operations Research. PrenticeHall, Englewood Cliffs, NJ.

Welch, J. S. 1987. PAM-A practitioners' approach to modeling. Management Sci. 33(5) 610-625. 